Portland State University

PDXScholar

Electrical and Computer Engineering Faculty

Publications and Presentations

3-1-1998

\title{
Field Solutions for Bidirectional High-Gain Laser Amplifiers and Oscillators
}

Lee W. Casperson

Portland State University

Mohammad Azadeh

University of Washington - Seattle Campus

Follow this and additional works at: https://pdxscholar.library.pdx.edu/ece_fac

Part of the Electrical and Computer Engineering Commons

Let us know how access to this document benefits you.

\section{Citation Details}

Azadeh, M., \& Casperson, L. W. (1998). Field solutions for bidirectional high-gain laser amplifiers and oscillators. Journal Of Applied Physics, 83(5), 2399.

This Article is brought to you for free and open access. It has been accepted for inclusion in Electrical and Computer Engineering Faculty Publications and Presentations by an authorized administrator of PDXScholar. Please contact us if we can make this document more accessible: pdxscholar@pdx.edu. 


\title{
Field solutions for bidirectional high-gain laser amplifiers and oscillators
}

\author{
Mohammad Azadeh \\ Department of Electrical Engineering, University of Washington, Seattle, Washington 98195-2500 \\ Lee W. Casperson ${ }^{\text {a) }}$ \\ The Institute of Optics and The Rochester Theory Center for Optical Science and Engineering, University \\ of Rochester, Rochester, New York 14627-0186
}

(Received 8 September 1997; accepted for publication 11 November 1997)

\begin{abstract}
General analytical solutions are obtained for the amplitude, phase, and intensity of the electromagnetic waves in bidirectional homogeneously broadened high-gain laser amplifiers and oscillators. These solutions are important as increasingly high-gain lasers are being employed in practical systems. Expressions are derived relating the output power to the input, including the effects of arbitrary mirror reflectivities and frequency detunings from the line center. For negligible reflectivities, these regenerative amplifier results reduce to earlier expressions for single-pass high-gain amplifiers. Multivalued outputs also occur, and in the limit of low gain per pass the results are consistent with earlier studies of single-frequency laser oscillators. (C) 1998 American Institute of Physics. [S0021-8979(98)00305-3]
\end{abstract}

\section{INTRODUCTION}

One of the most basic problems in optoelectronics is the calculation of the output power of saturating laser amplifiers, and such devices are used in many applications. For instance, on the large scale, optical amplifiers are the basis for the highest-powered fusion and weapons systems, while on the smaller side they have also attracted attention for their potential use in all optical signal processing and communication systems. Such wide-ranging usage underlines the value of having analytic models for these amplifiers. Many such models involving various degrees of approximation have been developed to study the characteristics of laser amplifiers and oscillators. ${ }^{1-5}$

Among the important difficulties in modeling many laser oscillators are the high gain of the laser medium and the low reflectivity of the mirrors. These properties may make it necessary to employ spatially dependent models for the electromagnetic fields and for the saturating laser medium. However, the most popular analytical models for laser oscillators employ an average intensity or photon density inside the amplifier. This approximation is not justified when the gain of the amplifier is so high that the sum of the right and left traveling intensities cannot be approximated with a constant value. On the other hand, in laser amplifiers the inevitable small reflectivity of even antireflection coated surfaces may also make it necessary to include bidirectional effects in the analysis of what otherwise would be unidirectional systems. When such reflections are significant, these systems should sometimes be regarded as regenerative laser amplifiers, and amplifier gain and efficiency may be increased substantially through multipass regeneration. ${ }^{6}$ Useful bistability and

\footnotetext{
${ }^{a)}$ Permanent address: Department of Electrical Engineering, Portland State
} University, P.O. Box 751, Portland, Oregon 97207-0751. switching behaviors can also occur in amplifier systems with appropriate feedback.

In many high-gain laser-amplifier systems, feedback is considered to be undesirable, and in the worst cases prelasing and parasitic oscillations may drain away pump energy that would otherwise have contributed to the useful laser output. Several techniques have been developed to reduce such unwanted feedback. Thus, the ends of the laser media may be cut at an oblique angle to the rod axis, often at the Brewster angle, or they are coated with antireflection layers. ${ }^{7}$ Reflections of off-axis spontaneous emission from the amplifier sides may be minimized by the use of black coatings, indexmatching fluids, or rough grinding of the sides. If more than one stage of amplification is required, the stages may be spaced as widely as possible, or they may be separated by electro-optical shutters, isolators, or bleachable media. Whether the feedback occurring in an amplifier system is desirable or not, its potential consequences should always be understood.

In this study, we develop a model for the behavior of a regenerative laser amplifier that consists of a general homogeneously broadened amplifying medium situated between two mirrors. We do not make the average intensity approximation, and thus we allow for arbitrarily high-gain values per pass and arbitrary reflectivities. Such generality is needed for the most useful analysis of laser amplifiers and oscillators. If the gain coefficient is considered to be negative, the results are also applicable to absorbing media having reflecting boundaries or situated between mirrors.

Another subject, which we address explicitly, is the phase of the fields inside the active medium. Most singlepass and regenerative amplifier models include only intensities, neglecting the phase. We show that the amplitude and phase of the fields can be related to each other by a simple expression, without making any significant approximations. Thus, once the amplitude of the wave is calculated, the phase 
of the wave can readily be found as well. The amplifier model we develop includes the effects of cavity frequency detunings from the line center and saturation-dependent dispersion, and the results reduce to known special cases. Among these special cases are the intensity transfer in a mirrorless laser amplifier, the intensity output and oscillation frequency of a laser oscillator, and the multivaluedness of the output of a laser above threshold as a function of the input intensity. Only the low-gain limiting case of this last effect has been considered previously. ${ }^{8}$

The basic equations for the electromagnetic fields in a saturating homogeneously broadened amplifying medium are developed in Sec. II. A general relation between the amplitude and phase of the field is derived in Sec. III. The equations are integrated in Sec. IV, and they are shown in Sec. V to reduce to familiar results for a laser amplifier when the reflectivities are set equal to zero. In Sec. VI they are shown to reduce to conventional oscillator formulas for the case of low gain per pass. Solutions for the general regenerative amplifier configuration are reported in Sec. VII.

\section{FIELD AND INTENSITY EQUATIONS}

The starting point in any derivation of electromagnetic wave propagation is Maxwell's equations. For a travelingwave laser amplifier, one can assume a propagating-wave form for the electric field

$$
E(z, t)=\frac{1}{2} E^{\prime}(z) \exp (i k z-i \omega t)+\text { c.c. },
$$

where $E(z, t)$ is the magnitude of the electric-field vector (which is assumed to be polarized in the $x$ direction), $E^{\prime}(z)$ is the slowly varying complex amplitude, and c.c. represents the complex conjugate. Assuming a similar form for the polarization, and after making the usual derivative approximation, ${ }^{9}$ one obtains

$$
\begin{gathered}
c_{1} \frac{d E^{\prime}(z)}{d z}+\frac{\gamma_{c} \omega}{\Omega} E^{\prime}(z)-i \frac{\left(\omega^{2}-\Omega^{2}\right)}{2 \Omega} E^{\prime}(z) \\
=i \frac{\omega^{2}}{2 \epsilon_{1} \Omega} \int_{0}^{\infty} \int_{-\infty}^{\infty} P^{\prime}\left(V, \omega_{\alpha}, z\right) d v d \omega_{\alpha},
\end{gathered}
$$

where $P^{\prime}\left(v, \omega_{\alpha}, z\right)$ is the complex amplitude of the polarization as a function of the $z$ component of the velocity of the active atoms or molecules, $\omega_{\alpha}$ is the center frequency of the laser transition for members of an atomic or molecular class $\alpha$, and $z$ is the position in the amplifier. The background speed of light in the medium characterized by the permeability $\mu_{1}$ and permittivity $\epsilon_{1}$ is $c_{1}=\left(\mu_{1} \epsilon_{1}\right)^{-1 / 2}, \gamma_{c}=\sigma / 2 \epsilon_{1}$ is the field decay rate, and $\Omega=k\left(\mu_{1} \epsilon_{1}\right)^{-1 / 2}$ can be recognized as the optical frequency at which the real part of the polarization vanishes. It is also possible to relate the polarization term back to the electric field, and for the simple case of a homogeneously broadened material with $\Omega=\omega$, the field equation can be written ${ }^{10}$

$$
\frac{d A(z)}{d z}=-\frac{n \gamma_{c}}{c}\left[A(z)-\frac{(1+i y) D_{0}(z) A(z)}{1+y^{2}+|A(z)|^{2}}\right]
$$

where $A(z)$ is a normalized field amplitude given by

$$
A(z)=\left(\frac{\gamma_{a}-\gamma_{a b}+\gamma_{b}}{2 \gamma \gamma_{a} \gamma_{b}}\right)^{1 / 2} \frac{\mu E^{\prime}(z)}{\hbar},
$$

the normalized optical frequency is $y=\left(\omega-\omega_{0}\right) / \gamma$ with $\omega_{0}$ the center frequency of the transition, $D_{0}(z)$ is the normalized unsaturated population difference, $\mu$ is the dipole moment of the transition, $\gamma_{a}$ and $\gamma_{b}$ are the total decay rates for the upper and lower laser levels, $\gamma_{a b}$ is the rate of direct decays from the upper level to the lower level, and $\gamma$ is the decay rate for the off-diagonal elements of the density matrix. In Eq. (3) $n=c / c_{1}$ is the background index of refraction, and the coefficient $n \gamma_{c} / c$ corresponds to a loss rate per unit of propagation distance.

In a standing-wave laser amplifier, one readily finds that the right and left traveling waves obey equations similar to Eq. (3), except that the saturation terms in these equations include an oscillating interference term as well as the sum of the left and right traveling intensities. Thus, the equations for the right and left traveling waves can be written

$$
\begin{aligned}
& \frac{d A^{+}(z)}{d z}=-\frac{n \gamma_{c}}{c}\left\{A^{+}(z)-\frac{(1+i y) D_{0}(z) A^{+}(z)}{1+y^{2}+\left[\left|A^{+}(z)\right|^{2}+\left|A^{-}(z)\right|^{2}+2\left|A^{+}(z) A^{-}(z)\right| \cos (2 k z)\right]}\right\}, \\
& \frac{d A^{-}(z)}{d z}=+\frac{n \gamma_{c}}{c}\left\{A^{-}(z)-\frac{(1+i y) D_{0}(z) A^{-}(z)}{1+y^{2}+\left[\left|A^{+}(z)\right|^{2}+\left|A^{-}(z)\right|^{2}+2\left|A^{+}(z) A^{-}(z)\right| \cos (2 k z)\right]}\right\},
\end{aligned}
$$

where $A^{+}(z)$ and $A^{-}(z)$ are the normalized complex amplitudes of the right and left traveling waves at a point $z$ inside the amplifier, and the relative phases of the right and left waves have been assumed to be unimportant (they could be included by shifting the arguments of the cos functions). These equations are quite general and account for the effects of line center detuning, longitudinal hole burning, and the possibility of high gain per pass.

The above equations govern the spatial evolution of the electromagnetic fields. It is also useful to convert them to an equivalent set of equations for the right and left traveling intensities. By defining normalized intensities $s I^{+}(z)=\left|A^{+}(z)\right|^{2}$ and $s I^{-}=\left|A^{-}(z)\right|^{2}$, one can obtain from Eqs. (5) and (6) 


$$
\begin{aligned}
& \frac{d I^{+}(z)}{d z}=-\frac{2 n \gamma_{c}}{c}\left(I^{+}(z)-\frac{D_{0}(z) I^{+}(z)}{1+y^{2}+s\left\{I^{+}(z)+I^{-}(z)+2\left[I^{+}(z) I^{-}(z)\right]^{1 / 2} \cos (2 k z)\right\}}\right), \\
& \frac{d I^{-}(z)}{d z}=+\frac{2 n \gamma_{c}}{c}\left(I^{-}(z)-\frac{D_{0}(z) I^{-}(z)}{1+y^{2}+s\left\{I^{+}(z)+I^{-}(z)+2\left[I^{+}(z) I^{-}(z)\right]^{1 / 2} \cos (2 k z)\right\}}\right) .
\end{aligned}
$$

In this notation $I^{+}$and $I^{-}$are the actual intensities and $s$ is a saturation parameter. It should be noticed that although the set of Eqs. (5) and (6) closely resemble Eqs. (7) and (8), in going from the former to the latter, information on the phase of the waves has been lost.

\section{PHASE OF THE FIELD IN A BIDIRECTIONAL AMPLIFIER}

Our first goal is to find a relationship between the phase and the amplitude of the electric field in a bidirectional laser amplifier. It would then be sufficient to solve the intensity equations for each particular case without losing the generality of the problem, because once the intensity or amplitude distribution has been found the phase distribution is automatically known. To achieve this goal, one can start from Eqs. (5) and (6). The right-hand sides of these two equations differ only by a sign, and one can easily deduce the following relationship

$$
\frac{1}{A^{+}(z)} \frac{d A^{+}(z)}{d z}+\frac{1}{A^{-}(z)} \frac{d A^{-}(z)}{d z}=0 .
$$

From this result follow the constraints $A^{+} A^{-}=$constant $\equiv s \quad a$ and $\left|A^{+}\right|^{2}\left|A^{-}\right|^{2}=s^{2} I^{+} I^{-}=s^{2}|a|^{2}$, where the constant $a$ has been introduced. This constant is allowed to be a complex quantity, unlike previous studies where the corresponding parameter has always been assumed to be real. ${ }^{1-3}$ It follows from these constraints that the right and left traveling complex amplitudes can be written in the form

$$
\begin{aligned}
& A^{+}(z)=s^{1 / 2} a^{1 / 2} \exp [\alpha(z)+i \beta(z)], \\
& A^{-}(z)=s^{1 / 2} a^{1 / 2} \exp [-\alpha(z)-i \beta(z)] .
\end{aligned}
$$

In these expressions, $\alpha(z)$ and $\beta(z)$ are new (real) functions of $z$, as yet unknown.

Equations (10) and (11) can now be substituted back into the field Eqs. (5) and (6). For example, if one substitutes Eq. (10) into Eq. (5) and cancels the exponential terms, one obtains two equations for the real and imaginary parts:

$$
\begin{aligned}
& \frac{d \alpha(z)}{d z}=-\frac{n \gamma_{c}}{c}\left(1-\frac{D_{0}(z)}{1+y^{2}+s|a|\{\exp [2 \alpha(z)]+\exp [-2 \alpha(z)]+2 \cos (2 k z)\}}\right), \\
& \frac{d \beta(z)}{d z}=\frac{n \gamma_{c}}{c} \frac{y D_{0}(z)}{1+y^{2}+s|a|\{\exp [2 \alpha(z)]+\exp [-2 \alpha(z)]+2 \cos (2 k z)\}} .
\end{aligned}
$$

Equations (12) and (13) can be combined to get a simple relationship between the amplitude and phase of the electric fields. The result is

$$
\frac{d \beta(z)}{d z}=y\left[\frac{d \alpha(z)}{d z}+\frac{n \gamma_{c}}{c}\right],
$$

which can be integrated to obtain

$$
\beta(z)=y \alpha(z)+y \frac{n \gamma_{c}}{c} z+\phi
$$

where $\phi$ is an integration constant.

Equation (15) is a simple but powerful result, since it relates the amplitude and the phase of the electric field in any bidirectional laser amplifier or oscillator. Therefore, from Eqs. (10) and (11) the complex electric-field amplitudes now can be written in terms of only one variable in the form

$$
\begin{aligned}
& A^{+}(z)=s^{1 / 2} a^{1 / 2} \exp \left[(1+i y) \alpha(z)+i y \frac{n \gamma_{c}}{c} z+i \phi\right], \\
& A^{-}(z)=s^{1 / 2} a^{1 / 2} \exp \left[-(1+i y) \alpha(z)-i y \frac{n \gamma_{c}}{c} z-i \phi\right] .
\end{aligned}
$$

These equations are not complete solutions yet. In fact, they still include two unknowns - the constant $a$, which may be calculated using boundary conditions, and the function $\alpha(z)$, which is the solution to Eq. (12).

\section{INTEGRATION OF THE WAVE EQUATION}

In the previous section it was shown that it would be sufficient to integrate the intensity wave equations in a bidirectional laser, since there is a simple relationship between the amplitude and the phase of the fields. Unfortunately, the 


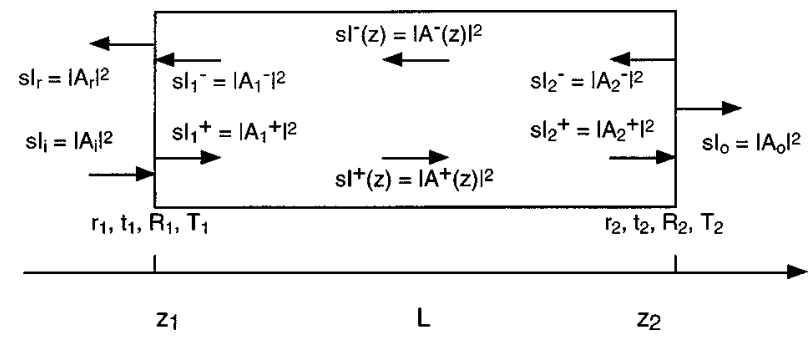

FIG. 1. Definition of intensity, field, and boundary variables in a laser amplifier.

more general form of the wave equations [any of Eqs. (5) and (6), (7) and (8), or (12)] cannot be integrated analytically, and one needs to make further approximations.

In many practical cases, the effects of the longitudinal spatial hole burning can be neglected by arguing that the oscillating term $\cos (2 \mathrm{kz})$ in the denominator of Eqs. (5) and (6), (7) and (8), or (12) is significant only in the short distance within a high-gain amplifier where the magnitudes of the right and left waves are about the same. Alternatively, one may often argue that spatial cross relaxation causes this term to average to zero. ${ }^{11}$ Neglecting longitudinal spatial hole burning makes the intensity wave equation analytically solvable, but the results are still very complicated. Another possible simplification is the neglect of the distributed loss term, the first term on the right-hand side of Eqs. (5) - (8) and (12). Although this simplification is not necessary, ${ }^{2,4,5}$ it is appropriate for many practical laser amplifiers, and it makes the results much less complicated. With these two approximations, one can reduce Eqs. (5), (7), and (12) to the following equations, respectively,

$$
\begin{aligned}
& \frac{d A^{+}(z)}{d z}=\frac{n \gamma_{c}}{c} \frac{(1+i y) D_{0}(z) A^{+}(z)}{1+y^{2}+\left[\left|A^{+}(z)\right|^{2}+s^{2}|a|^{2} /\left|A^{+}(z)\right|^{2}\right]}, \\
& \frac{d I^{+}(z)}{d z}=\frac{n \gamma_{c}}{c} \frac{2 D_{0}(z) I^{+}(z)}{1+y^{2}+s\left[I^{+}(z)+|a|^{2} / I^{+}(z)\right]}, \\
& \frac{d \alpha(z)}{d z}=\frac{n \gamma_{c}}{c} \frac{D_{0}(z)}{1+y^{2}+s|a|\{\exp [2 \alpha(z)]+\exp [-2 \alpha(z)]\}},
\end{aligned}
$$

where in Eqs. (18) and (19) the relationship following Eq. (9) has been used. The first two of the above equations are for the right traveling waves and the equations for the left traveling waves are similar. We will now assume that the unsaturated population difference $D_{0}$ is independent of position $z$, and thus, Eqs. (19) and (20) are now in an integrable form. The corresponding phase information can readily be obtained by application of Eq. (15) once the intensity or amplitude has been solved. Therefore, we now concentrate on the intensity wave equations.

By introducing the new gain and saturation parameters

$$
\begin{aligned}
& g=\frac{n \gamma_{c}}{c} \frac{2 D_{0}}{1+y^{2}}, \\
& s^{\prime}=\frac{s}{1+y^{2}},
\end{aligned}
$$

Eq. (19) can be written in the more compact form

$$
\frac{d I^{+}(z)}{d z}=\frac{g I^{+}(z)}{1+s^{\prime}\left[I^{+}(z)+|a|^{2} / I^{+}(z)\right]} .
$$

This equation can be integrated and the result is

$s^{\prime}\left[I^{+}\left(z_{2}\right)-I^{+}\left(z_{1}\right)\right]+\ln \left[\frac{I^{+}\left(z_{2}\right)}{I^{+}\left(z_{1}\right)}\right]-\frac{s^{\prime}|a|^{2}}{I^{+}\left(z_{2}\right)}+\frac{s^{\prime}|a|^{2}}{I^{+}\left(z_{1}\right)}=g L$.

The above expression relates the positive traveling intensity at the two ends of the amplifier, the gain-length product $g L$, and the modulus of the parameter $a$. On the other hand, the quantities $I^{+}\left(z_{1}\right)$ and $I^{+}\left(z_{2}\right)$ can be related to the amplifier input and output via appropriate boundary conditions. It is, therefore, useful to solve Eq. (24) for the quantity $|a|^{2}$, since this quantity should eventually be eliminated from the equations. Moreover, we previously expressed the electric field in terms of the two new functions $\alpha(z)$ and $\beta(z)$ representing the amplitude and the phase of the field, respectively, so the intensity of the waves can be expressed in terms of the function $\alpha(z)$ only. In fact, it can be seen from Eqs. (16) and (17) that

$$
\begin{aligned}
& s I^{+}(z)=\left|A^{+}(z)\right|^{2}=s|a| \exp [2 \alpha(z)], \\
& s I^{-}(z)=\left|A^{-}(z)\right|^{2}=s|a| \exp [-2 \alpha(z)] .
\end{aligned}
$$

Therefore, expressing $I^{+}\left(z_{1}\right)$ and $I^{+}\left(z_{2}\right)$ in terms of $\alpha\left(z_{1}\right)$ and $\alpha\left(z_{2}\right)$ and solving for $|a|$, one obtains from Eq. (24)

$$
|a|=\frac{g L-2 \alpha\left(z_{2}\right)+2 \alpha\left(z_{1}\right)}{s^{\prime} \exp \left[2 \alpha\left(z_{2}\right)\right]-s^{\prime} \exp \left[2 \alpha\left(z_{1}\right)\right]-s^{\prime} \exp \left[-2 \alpha\left(z_{2}\right)\right]+s^{\prime} \exp \left[-2 \alpha\left(z_{1}\right)\right]} .
$$

Equation (27) is the solution of the wave equation in the amplifier. However, the quantities $|a|, \alpha\left(z_{1}\right)$, and $\alpha\left(z_{2}\right)$ should still be related to the amplifier input and output intensities by the appropriate boundary conditions.

Referring to Fig. 1, the boundary conditions at the input and output side of an amplifier can be written as

$$
\begin{aligned}
& I_{1}^{+}=R_{1} I_{1}^{-}+T_{1} I_{i}, \\
& I_{2}^{-}=R_{2} I_{2}^{+}, \\
& I_{0}=T_{2} I_{2}^{+},
\end{aligned}
$$


where $T_{1}, T_{2}, R_{1}$, and $R_{2}$ are the intensity transmission and reflection coefficients at the input and output sides of the amplifier. There is also an output at the left-hand end of the amplifier, but to be specific we are emphasizing the output on the right. Now, we can use Eqs. (25) and (26) and write the first two of the above boundary conditions in terms of the variables $\alpha\left(z_{1}\right)$ and $\alpha\left(z_{2}\right)$ :

$$
\begin{aligned}
& |a| \exp \left[2 \alpha\left(z_{1}\right)\right]=R_{1}|a| \exp \left[-2 \alpha\left(z_{1}\right)\right]+T_{1} I_{i}, \\
& |a| \exp \left[-2 \alpha\left(z_{2}\right)\right]=R_{2}|a| \exp \left[2 \alpha\left(z_{2}\right)\right] .
\end{aligned}
$$

Solving for $\alpha\left(z_{1}\right)$ and $\alpha\left(z_{2}\right)$ one obtains

$$
\begin{aligned}
& \alpha\left(z_{1}\right)=\frac{1}{2} \ln \left[\frac{T_{1} I_{i}+\left(T_{1}^{2} I_{i}^{2}+4 R_{1}|a|^{2}\right)^{1 / 2}}{2|a|}\right], \\
& \alpha\left(z_{2}\right)=\frac{1}{2} \ln \left[\frac{1}{\left(R_{2}\right)^{1 / 2}}\right] .
\end{aligned}
$$

In solving the above quadratic equations, the positive root has been chosen because the argument of the logarithm functions should always be positive $\left[\alpha\left(z_{1}\right)\right.$ and $\alpha\left(z_{2}\right)$ should be real].

By multiplying both sides of Eq. (29) by $I_{2}^{+}$and noticing that $I_{0}=T_{2} I_{2}^{+}$, one may obtain

$$
|a|^{2}=R_{2}\left(\frac{I_{0}}{T_{2}}\right)^{2} .
$$

We can now combine the above equations, which include boundary conditions for input and output intensities with the results of the integration of the wave equation. For an optical cavity with the parameters defined in Fig. 1, one has

$$
\frac{A_{1}^{+}}{A_{i}}=\frac{t_{1}}{1-G_{\text {r.t. }}},
$$

where $t_{1}$ and $G_{\text {r.t. }}$ are the complex field transmission coefficient at the input side and the complex round-trip gain. Equation (36) can easily be extended to relate the input and output fields:

$$
\frac{A_{0}}{A_{i}}=\frac{t_{1} t_{2} G_{\text {s.p. }}}{1-G_{\text {r.t. }}},
$$

where the single-pass gain $G_{\text {s.p. }}=A^{+}\left(z_{2}\right) \exp \left(i k z_{2}\right) /$ $A^{+}\left(z_{1}\right) \exp \left(i k z_{1}\right)$ is the complex gain from the input side to the output side inside the cavity and $t_{2}$ is the complex fieldtransmission coefficient at the output side of the amplifier.

The above expressions are commonly used for a linear Fabry-Perot étalon. However, one can extend them to include the saturation and detuning effects in an amplifier. To do this, we need to express the single-pass and round-trip gains in terms of the results of the previous section, i.e., in terms of the function $\alpha(z)$. Making use of Eqs. (16) and (17), and assuming again that the distributed loss is small compared to the gain, we obtain

$$
\begin{aligned}
G_{\text {s.p. }} & =\frac{A^{+}\left(z_{2}\right) \exp \left(i k z_{2}\right)}{A^{+}\left(z_{1}\right) \exp \left(i k z_{1}\right)} \\
& =\exp \left\{(1+i y)\left[\alpha\left(z_{2}\right)-\alpha\left(z_{1}\right)\right]+i \frac{n \omega}{c} L\right\}, \\
G_{\text {r.t. }} & =\frac{A^{+}\left(z_{2}\right) \exp \left(i k z_{2}\right)}{A^{+}\left(z_{1}\right) \exp \left(i k z_{1}\right)} \times r_{2} \times \frac{A^{-}\left(z_{1}\right) \exp \left(i k z_{1}\right)}{A^{-}\left(z_{2}\right) \exp \left(i k z_{2}\right)} \times r_{1} \\
& =r_{1} r_{2} \exp \left\{2(1+i y)\left[\alpha\left(z_{2}\right)-\alpha\left(z_{1}\right)\right]+2 i \frac{n \omega}{c} L\right\},
\end{aligned}
$$

where we have used $L=z_{2}-z_{1}$ and $k=n \omega / c$. It is evident that these expressions for the round-trip and single-pass gain are in terms of the quantity $\alpha\left(z_{2}\right)-\alpha\left(z_{1}\right)$. If we define this quantity as the new parameter

$$
H=\alpha\left(z_{2}\right)-\alpha\left(z_{1}\right),
$$

then Eq. (37) can be written as

$$
\frac{A_{0}}{A_{i}}=\frac{t_{1} t_{2} \exp \left[(1+i y) H+i \frac{n \omega}{c} L\right]}{1-r_{1} r_{2} \exp \left[2(1+i y) H+2 i \frac{n \omega}{c} L\right]} .
$$

Equation (41) can also be expressed in terms of the intensities. It can readily be verified that

$$
\frac{I_{0}}{I_{i}}=\left|\frac{A_{0}}{A_{i}}\right|^{2}=\frac{T_{1} T_{2} \exp (2 H)}{\left[1-\left(R_{1} R_{2}\right)^{1 / 2} \exp (2 H)\right]^{2}+4\left(R_{1} R_{2}\right)^{1 / 2} \exp (2 H) \sin ^{2}\left[y H+\frac{n \omega}{c} L+\frac{\theta_{1}+\theta_{2}}{2}\right]},
$$

where $\theta_{1}$ and $\theta_{2}$ are the phase shifts for reflection at the boundaries. The parameter $H$ is intrinsically related to the saturating gain in the amplifier. For a gainless medium, the intensities at both sides of the amplifier are equal $\left[I^{+}\left(z_{2}\right)=I^{+}\left(z_{1}\right)\right]$, and therefore, $H=0$. In this case, Eq. (42) reduces to

$$
\frac{I_{0}}{I_{i}}=\left|\frac{A_{0}}{A_{i}}\right|^{2}=\frac{T_{1} T_{2}}{\left[1-\left(R_{1} R_{2}\right)^{1 / 2}\right]^{2}+4\left(R_{1} R_{2}\right)^{1 / 2} \sin ^{2}\left[\frac{n \omega}{c} L+\frac{\theta_{1}+\theta_{2}}{2}\right]},
$$

which is a well-known expression for the power transmission coefficient of a Fabry-Perot étalon.

The more interesting case is when $H$ is not zero, where Eq. (42) becomes nonlinear since $H$ itself would be a function of the intensities. To calculate $H$ in this case, one can make use of Eqs. (27), (33), (34), and (35) to obtain 


$$
\begin{aligned}
& H=\alpha\left(z_{2}\right)-\alpha\left(z_{1}\right)=s^{\prime} \frac{R_{2}^{1 / 2}}{2 T_{2}} \\
& \times I_{0}\left\{R_{2}^{1 / 2}-\frac{1}{R_{2}^{1 / 2}}+\frac{T_{1} I_{i}+\left[T_{1}^{2} I_{i}^{2}+4 R_{1} R_{2}\left(\frac{I_{0}}{T_{2}}\right)^{2}\right]^{1 / 2}}{\frac{2 R_{2}^{1 / 2}}{T_{2}} I_{0}}-\frac{\frac{2 R_{2}^{1 / 2}}{T_{2}} I_{0}}{T_{1} I_{i}+\left[T_{1}^{2} I_{i}^{2}+4 R_{1} R_{2}\left(\frac{I_{0}}{T_{2}}\right)^{2}\right]^{1 / 2}}\right\}+\frac{g L}{2} .
\end{aligned}
$$

Therefore, in principle, one can substitute Eq. (44) into Eq. (42) and obtain the intensity gain coefficient for a saturating bidirectional amplifier.

The above equation simplifies slightly if we introduce normalized input and output intensities

$$
\begin{aligned}
& J_{i}=T_{1} s^{\prime} I_{i}, \\
& J_{0}=\frac{2\left(R_{1} R_{2}\right)^{1 / 2}}{T_{2}} s^{\prime} I_{0} .
\end{aligned}
$$

Using the above-normalized intensities and also defining $R^{2}=R_{1} R_{2}$, Eqs. (42) and (44) become

$$
\frac{J_{0}}{J_{i}}=\frac{2 R \exp (2 H)}{[1-R \exp (2 H)]^{2}+4 R \exp (2 H) \sin ^{2}\left[y H+\frac{n \omega}{c} L+\frac{\theta_{1}+\theta_{2}}{2}\right]},
$$

$$
\begin{aligned}
H= & \frac{J_{0}}{4}\left[\frac{R}{R_{1}}-\frac{1}{R}+\frac{J_{i}+\left(J_{i}^{2}+J_{0}^{2}\right)^{1 / 2}}{J_{0}}\right. \\
& \left.-\frac{1}{R_{1}} \frac{J_{0}}{J_{i}+\left(J_{i}^{2}+J_{0}^{2}\right)^{1 / 2}}\right]+\frac{g L}{2} .
\end{aligned}
$$

These two equations allow for calculation of the output of a high-gain amplifier for a given input, including saturation and detuning effects. They should have many applications in the study of high-gain laser systems, including those with non-negligible end reflectivities. In the remainder of this study, we consider several special cases of these equations.

\section{SINGLE-PASS AMPLIFIER}

Certain special cases of our general amplifier formulas correspond to previously known results. As a first example, one may consider the situation in which the feedback in the bidirectional amplifier is reduced to zero. In this case, the input-output relation should be the same as for a basic single-pass amplifier.

If the reflectivity $R_{2}$ of the output mirror becomes small, Eq. (42) reduces to

$$
\frac{I_{0}}{I_{i}}=T_{1} T_{2} \exp (2 H)
$$

and Eq. (44) reduces to

$$
\begin{aligned}
H & =s^{\prime} \frac{R_{2}^{1 / 2}}{2 T_{2}} I_{0}\left(-\frac{1}{R_{2}^{1 / 2}}+\frac{T_{1} T_{2} I_{i}}{R_{2}^{1 / 2} I_{0}}\right)+\frac{g L}{2} \\
& =\frac{s^{\prime}}{2 T_{2}}\left(T_{1} T_{2} I_{i}-I_{0}\right)+\frac{g L}{2} .
\end{aligned}
$$

These equations may be combined to yield

$$
\frac{I_{0}}{I_{i}}=T_{1} T_{2} \exp \left[\frac{s^{\prime}}{T_{2}}\left(T_{1} T_{2} I_{i}-I_{0}\right)+g L\right],
$$

a simpler transcendental relationship between the input and output intensities.

In an ideal amplifier, there would also be no loss at the ends of the amplifying region. Setting $T_{1}=T_{2}=1$, Eq. (51) can be written in the form

$$
s^{\prime} I_{0}-s^{\prime} I_{i}+\ln \left(\frac{s^{\prime} I_{0}}{s^{\prime} I_{i}}\right)=g L .
$$

If the input signal is at the center frequency of the transition so that $s^{\prime}$ and $g$ have their line-center values, then Eq. (52) can be recognized as the standard input-output relation for a homogeneously broadened laser. ${ }^{12,13}$

\section{LASER OSCILLATOR}

A laser oscillator above threshold can be considered as a laser amplifier with no input. In this case, the frequency of oscillation and the output intensity are determined by the cavity itself. The oscillation condition requires the complex round-trip gain to be unity [this can be seen by setting the denominator of Eq. (41) to zero], which by itself amounts to two conditions: the magnitude of the round-trip gain should be unity and the round-trip phase delay should be an integral multiple of $2 \pi$. The former condition determines the output intensity of the laser, while the latter condition determines the frequency of oscillation. From Eq. (42), the gain condition is

$$
\left(R_{1} R_{2}\right)^{1 / 2} \exp (2 H)=1 .
$$

On the other hand, Eq. (48) in this case simplifies to (by setting $J_{i}$ to zero) 


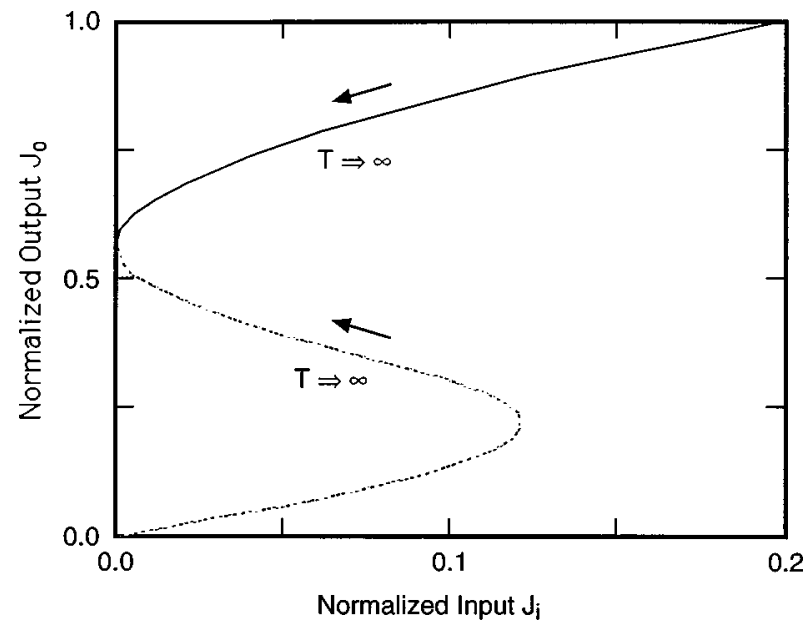

FIG. 2. Normalized amplifier output intensity vs normalized input intensity with zero detuning and a gain-length product of $g L=2.5$. Other parameters are $R_{1}=R_{2}=0.3$, and $T_{1}=T_{2}=0.7$.

$$
H=\frac{J_{0}}{4}\left(\frac{R}{R_{1}}-\frac{1}{R}+1-\frac{1}{R_{1}}\right)+\frac{g L}{2} .
$$

Combining Eqs. (46), (53), and (54), one obtains

$$
I_{0}=\frac{T_{2}}{s^{\prime}} \frac{g L+\ln \left(R_{1} R_{2}\right)^{1 / 2}}{1-R_{2}+\left(R_{2} / R_{1}\right)^{1 / 2}-\left(R_{1} R_{2}\right)^{1 / 2}},
$$

which is a known result for the output intensity of a laser oscillator. $^{1}$

The condition that the round-trip phase be an integral multiply of $2 \pi$ determines the frequency of oscillation. Using Eq. (42), this condition becomes

$$
2 y H+2 \frac{n \omega}{c} L+\theta_{1}+\theta_{2}=2 m \pi,
$$

where $m$ is an integer. One can combine this result with Eq. (53) and solve for the oscillation frequency:

$$
\omega=\frac{\left(2 m \pi-\theta_{1}-\theta_{2}\right) \gamma c-\omega_{0} c \ln \left(R_{1} R_{2}\right)^{1 / 2}}{2 \gamma n L-c \ln \left(R_{1} R_{2}\right)^{1 / 2}} .
$$

This useful equation describes the $m$ th cavity mode oscillation frequency of a high-gain laser oscillator.

We should be able to reduce Eq. (57) to one of the well-known expressions for the oscillation frequency of a laser in some proper limit. To do this, we consider the lowgain per pass limit, where the reflectivities at the two sides are close to unity. We, therefore, assume that $R_{1}$ and $R_{2}$ are each less than unity by a small amount, which we call $\delta_{1}$ and $\delta_{2}$, respectively, $R_{1}=1-\delta_{1}, R_{2}=1-\delta_{2}$. In this case, the logarithm functions can be simplified, and Eq. (57) takes the following form:

$$
\omega=\frac{\left(2 m \pi-\theta_{1}-\theta_{2}\right) \gamma c+\left(\delta_{1}+\delta_{2}\right) c \omega_{0} / 2}{2 \gamma n L+\left(\delta_{1}+\delta_{2}\right) c / 2} .
$$

Moreover, in the limit of low gain-per-pass, it may be assumed that it takes many round trips before any significant

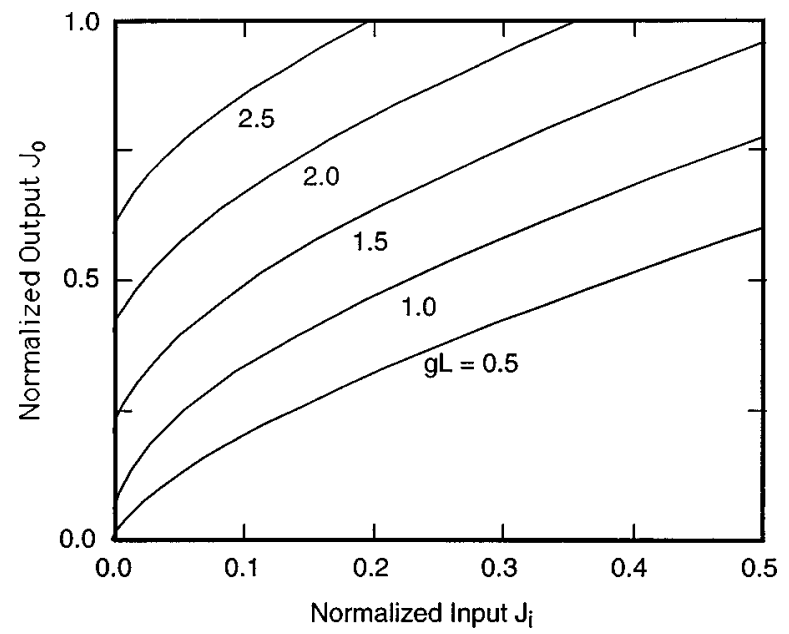

FIG. 3. Normalized amplifier output intensity vs normalized input intensity for various values of the gain-length product $g L$. Other parameters are as in Fig. 2.

change in the amplitude of the waves takes place. Hence, the losses at the boundaries may be averaged over the round-trip length of the amplifier, and the cavity lifetime may be defined as

$$
t_{c}=\frac{2 n L}{c\left(\delta_{1}+\delta_{2}\right)} .
$$

One can then define the cavity bandwidth $\Delta \nu_{c}=\left(2 \pi t_{c}\right)^{-1}$ and the $m$ th empty cavity mode frequency $\omega_{c}=\left[m \pi-\left(\theta_{1}\right.\right.$ $\left.\left.+\theta_{2}\right) / 2\right] c / n L$. Moreover, the atomic linewidth can be related to the decay rate $\gamma$ by $\gamma=\pi \Delta \nu_{h}$. Using these relations, Eq. (58) becomes

$$
\omega=\frac{\omega_{c} \Delta \nu_{h}+\omega_{0} \Delta \nu_{c}}{\Delta \nu_{h}+\Delta \nu_{c}},
$$

which is a well-known result. ${ }^{14,15}$ Therefore, the expressions we have obtained for a high-gain laser amplifier with feedback produce appropriate results when applied to the special case of a laser oscillator with no input.

\section{BIDIRECTIONAL AMPLIFIER}

In this section we consider the implications of Eqs. (47) and (48) when the input to the amplifier is not zero. In their most general form, these equations cannot be directly solved to obtain a single explicit expression for the output intensity of a laser amplifier as a function of the input intensity, although they can be plotted easily using a simple routine. On the other hand, in some simple cases these equations can be combined and the output can be expressed in a parametrized form, for example, as a function of the amplifier gain. We first start with the special cases where further analytical results may be obtained, and later consider the most general form of Eqs. (47) and (48). From Eq. (47) the round-trip phase-shift $\phi$ is found to be

$$
\phi=\frac{2 n L \omega}{c}+2 y H+\theta_{1}+\theta_{2} .
$$


For values of $\phi$ equal to an integral multiple of $2 \pi$, the amplifier is at resonance, i.e., a maximum transmission would take place. If the frequency of the input coincides with the center frequency of the atomic transition $(y=0)$, the round-trip phase shift would be constant for a given cavity, i.e., it would not be a function of input intensity. However, if $y$ is not zero, $\phi$ itself becomes intensity dependent via the parameter $H$. Hence, one should distinguish between the effects of detuning from the atomic line-center frequency and detuning from the cavity resonance.

For the special case of $y$ being zero, Eq. (47) can be solved for $H$

$H=\frac{1}{2} \ln \left\{\frac{J_{0} \cos \phi+J_{i} \pm\left[\left(J_{0} \cos \phi+J_{i}\right)^{2}-J_{0}^{2}\right]^{1 / 2}}{R J_{0}}\right\}$,

where $\phi$ is defined by Eq. (61) with $y=0$. The sign ambigu- ity in Eq. (62) is important and its implications will becomeclear shortly. Combining Eqs. (48) and (62), one obtains an implicit input-output equation

$$
\begin{gathered}
\ln \left\{\frac{J_{0} \cos \phi+J_{i} \pm\left[\left(J_{0} \cos \phi+J_{i}\right)^{2}-J_{0}^{2}\right]^{1 / 2}}{R J_{0}}\right\} \\
=\frac{J_{0}}{2}\left[\frac{R}{R_{1}}-\frac{1}{R}+\frac{J_{i}+\left(J_{i}^{2}+J_{0}^{2}\right)^{1 / 2}}{J_{0}}\right. \\
\left.-\frac{1}{R_{1}} \frac{J_{0}}{J_{i}+\left(J_{i}^{2}+J_{0}^{2}\right)^{1 / 2}}\right]+g L .
\end{gathered}
$$

This equation can be solved for the output intensity and parametrized in terms of the overall transmission coefficient $T=J_{0} / J_{i}$ :

$$
J_{0}=2 \frac{\ln \left\{T \cos \phi+1 \pm\left[(T \cos \phi+1)^{2}-T^{2}\right]^{1 / 2}\right\}-\ln (R T)-g L}{\frac{R}{R_{1}}-\frac{1}{R}+\frac{1+\left(1+T^{2}\right)^{1 / 2}}{T}-\frac{1}{R_{1}} \frac{T}{1+\left(1+T^{2}\right)^{1 / 2}}} .
$$

This result describes the output intensity from an amplifier with an intensity transmission gain of $T$. In this form, the intensity characteristics of the amplifier can easily be plotted either as a direct function of $T$ or by using $T$ as a parameter. It may be noted that Eq. (64) also reduces to Eq. (55) in the limiting case of a laser oscillator ( $T$ going to infinity and $\phi$ $=2 m \pi)$.

These results are best illustrated by means of a specific amplifier example. Figure 2 is a plot of the normalized output intensity versus the normalized input to an amplifier for a gain-length product $g L=2.5$, reflectivities $R_{1}=R_{2}=0.3$, transmission coefficients $T_{1}=T_{2}=0.7$, and round-trip phaseshift $\phi=2 m \pi$. The solid branch is the result of plotting Eq. (64) with the minus sign, and the dotted branch corresponds to Eq. (64) with the plus sign. The solid branch in Fig. 2 starts from a nonzero value for zero input (corresponding to a transmission gain $T$ of infinity) and describes the stable behavior of an amplifier above the threshold conditions, i.e., the amplifier may oscillate and produce an output even with no input. On the other hand, the dotted branch passes through the origin for low values of transmission gain $T$, and bends back to the vertical axis as $T$ approaches infinity. This branch may be considered unstable, since in reality it cannot be observed. This effect as well as other more detailed aspects of the stable and unstable branches have also been studied by Spencer and Lamb for a laser oscillator subject to an injected input. ${ }^{8}$

The transition from below-threshold to above-threshold conditions is depicted in Fig. 3, which shows the results of plotting the output power versus input (only the stable branch) using Eq. (64) for several values of the gain-length product $g L$. Other parameters are the same as in Fig. 2. It can be seen that for small values of $g L$, the input-output curve is almost linear. As $g L$ becomes larger, the differential gain close to the origin starts to increase and eventually becomes infinite (regenerative amplification). Further increase

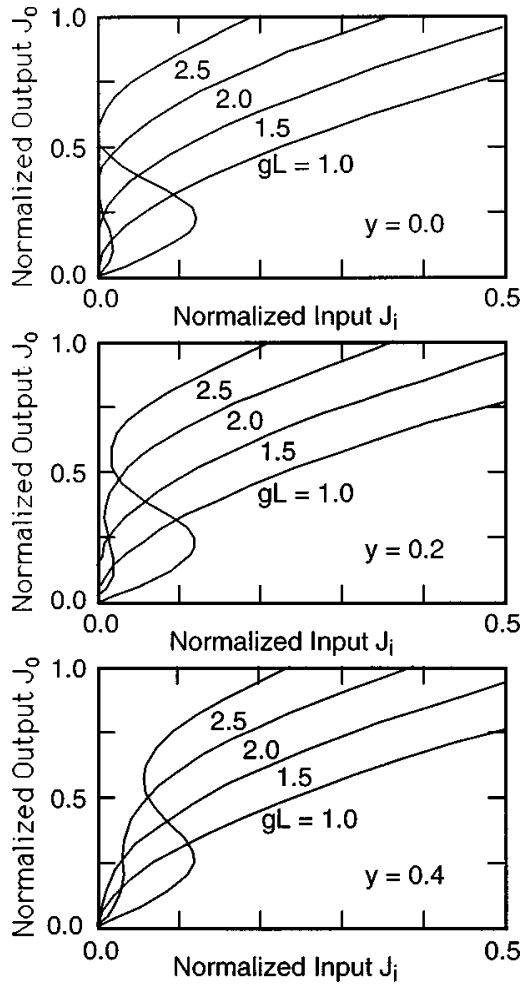

FIG. 4. Normalized amplifier output intensity vs normalized input intensity for various values of gain-length product and frequency detuning. 
in $g L$ causes oscillation, and the curves no longer pass through the origin corresponding to above-threshold conditions.

So far, we have only considered the case of zero linecenter and cavity detunings $(y=0, \phi=2 m \pi)$. As mentioned earlier, Eqs. (47) and (48) cannot be solved analytically when $y$ is not zero. This is because Eq. (47) cannot be solved for $H$, and the parametrization procedure used earlier cannot be carried out directly. However, it is still possible to plot the output intensity $J_{0}$ versus the input intensity $J_{i}$ using a simple numerical routine. To introduce this procedure, we write Eqs. (47) and (48) in the forms

$$
\begin{aligned}
& T(H)=\frac{2 R \exp (2 H)}{[1-R \exp (2 H)]^{2}+4 R \exp (2 H) \sin ^{2}\left(y H+\frac{n L \omega}{c}+\frac{\theta_{1}+\theta_{2}}{2}\right)}, \\
& J_{0}(H, T)=\frac{H-\frac{g L}{2}}{\frac{1}{4}\left[\frac{R}{R_{1}}-\frac{1}{R}+\frac{1+\left(1+T^{2}\right)^{1 / 2}}{T}-\frac{1}{R_{1}} \frac{T}{1+\left(1+T^{2}\right)^{1 / 2}}\right]} .
\end{aligned}
$$

Thus, if $H$ is taken as a parameter, then the transmission gain $T$ can be expressed as a function of $H$, and the output power $J_{0}$ can be expressed as a function of $H$ and $T$. Therefore, by using a simple routine we can plot the input-output curves in the most general case when the atomic frequency detuning $y$ is not zero. Moreover, this method of parametrization has the advantage of generating the stable and unstable branches at the same time.

Figure 4 illustrates the results of plotting the output of the amplifier versus the input for three values of line-center detuning $y=0,0.2$, and 0.4 , and for values of gain-length product ranging from 1 to 2.5 . Also, for simplicity we have set $2 n L \omega / c+\theta_{1}+\theta_{2}=2 m \pi$. The case of $y=0$ in Fig. 4 corresponds to Fig. 3, only now the unstable branches have been shown as well. It can also be seen that the unstable branches vanish if the gain-length product is not large enough. For values of $y$ not equal to zero, the curves no longer touch the vertical axis. In physical terms, this means that the input power has to reach a certain level before the amplifier is locked to the frequency of the input signal. Moreover, it takes a larger input level to lock the laser as the frequency is detuned further away from the center of the atomic transition. This behavior is again in agreement with the predictions of Spencer and $\mathrm{Lamb}^{8}$ for the special case of low-gain per pass.

\section{CONCLUSION}

Analytical solutions have been obtained for the fields in a homogenously broadened high-gain laser medium including a relationship between the phase and the amplitude of the waves in a bidirectional regenerative laser amplifier. By applying the appropriate boundary conditions, implicit equations have been found for the output power as a function of the input including detuning effects. When suitable limits are taken, these equations reduce to known results for the output intensity and the oscillation frequency of laser amplifiers and oscillators. When the input is not zero, bistability effects are found; and, for cases where the input is detuned from the center frequency of the atomic transition, no significant output is produced until the input reaches a critical level. Above this level, high-intensity oscillations may occur at a frequency that is locked to the input signal. The general results reported here should be useful for many applications of highgain laser systems having non-negligible end reflectivities.

\section{ACKNOWLEDGMENTS}

This work was supported in part by the National Science Foundation under Grant Nos. PHY94-15583 and ECS9014481. One of the authors (L.W.C.) would also like to express his appreciation to members of the Rochester Theory Center for Optical Science and Engineering and the Institute of Optics for valuable discussions and hospitality during his sabbatical visit.

${ }^{1}$ W. W. Rigrod, J. Appl. Phys. 36, 2487 (1965).

${ }^{2}$ W. W. Rigrod, IEEE J. Quantum Electron. QE-14, 377 (1978).

${ }^{3}$ L. W. Casperson, Appl. Opt. 19, 422 (1980).

${ }^{4}$ G. M. Schindler, IEEE J. Quantum Electron. QE-16, 546 (1980).

${ }^{5}$ A. M. Hunter II and R. O. Hunter, Jr., IEEE J. Quantum Electron. QE-17, 1879 (1981).

${ }^{6}$ D. Röss, in Lasers, Light Amplifiers and Oscillators (Academic, New York, 1969), pp. 90-99.

${ }^{7}$ W. Koechner, in Solid-State Laser Engineering (Springer, New York, 1976), pp. 166-168.

${ }^{8}$ M. B. Spencer and W. E. Lamb, Phys. Rev. A 5, 884 (1972).

${ }^{9}$ L. W. Casperson, Phys. Rev. A 44, 3291 (1991).

${ }^{10}$ Equations (60) and (61) of Ref. 9.

${ }^{11}$ H. G. Danielmeyer, J. Appl. Phys. 42, 3125 (1971).

${ }^{12}$ J. S. Wright and E. O. Schulz-DuBois, "Solid-State Maser Research," Fifth Quarterly Report, U.S. Army Signal Corps Contract No. DA36039SC-85357, 20 September 1961.

${ }^{13}$ W. W. Rigrod, J. Appl. Phys. 34, 2602 (1963).

${ }^{14} \mathrm{C}$. H. Townes, in Advances in Quantum Electronics, edited by J. R. Singer (Columbia University Press, New York, 1961), pp. 3-11.

${ }^{15}$ L. W. Casperson, Phys. Rev. A 42, 6721 (1990). 